Ensayo Original / Original Essay

\title{
Terapias Asistidas con Animales: Una Perspectiva de Protección Animal
}

\author{
Autores: Camilo Chacón Herrera \\ Tribunal Supremo de Justicia, TSJ \\ camilochacon2008@gmail.com \\ Aragua, Venezuela \\ Marian Serradas Fonseca \\ Universidad Nacional Abierta, UNA \\ mserradas@hotmail.com \\ Yaracuy, Venezuela
}

\section{Resumen}

Cuando se habla de terapias con animales, toda la literatura se vuelca en los beneficios del animal humano que participa de ellas, así como su comprobación científica, la óptica abarca también los posibles riesgos del humano con el contacto animal, inclusive los costos de dichas terapias, sin embargo, poca literatura analiza estas terapias con una óptica proteccionista del animal, siendo que es claro que en su mayoría, el afectado resulta siendo éste, pues suele ser apartado de su hábitat, sometido a entrenamientos crueles, a condiciones innaturales y de maltrato. El objetivo fundamental es visibilizar al animal como un ser vivo que siente, padece y que es merecedor de una serie de derechos que deben respetarse dentro de las terapias en que participa. Es por ello, que en esta ocasión, si bien la investigación parte de reconocer algunos beneficios de las terapias con animales, profundiza en aquellas características y condiciones que han de tener dichas terapias para que sean amigables con los animales que participan en ellas, constituyendo una lectura reflexiva y sensibilizadora, tanto para terapeutas, animalistas, pacientes y familiares. La revisión incluye un abordaje legal de la situación del maltrato animal y sus repercusiones en terapias con canes, delfines y equinos.

Palabras clave: terapia; derechos de los animales; recursos animales; animal doméstico; animal acuático. 


\title{
Animal-Assisted Therapies: An Animal Protection Perspective
}

\begin{abstract}
When talking about therapies with animals, alongside the scientific verification of the therapies, all the literature turns to the benefits of the human animal that participates in them. This viewpoint also covers the possible risks of animal contact for the human and the costs of such therapies. However, little literature analyzes these therapies with a protectionist view of the animal, although it is clear that the animal is often affected, since it is usually separated from its habitat, subjected to cruel trainings, to unnatural conditions and abuse. The fundamental objective is to make the animal visible as a living being that feels, suffers and deserves a series of rights that must be respected within the therapies in which it participates. On this occasion, although the research partly recognizes some benefits of animal therapies, it delves into the characteristics and conditions that these therapies must have in order to be friendly to the animals that participate in them. This constitutes a reflective and sensitive reading for therapists, animalists, patients and relatives alike. The review includes a legal approach to the situation of animal abuse and its repercussions in therapies with dogs, dolphins and horses.
\end{abstract}

Keywords: therapy; animal rights; animal resources; domestic animal; aquatic animal.

Date Received: 03-02-2018

Date Acceptance: 09-04-2018 
"Cuando exhortas el poder de un animal, estás pidiendo ser envuelto en armonía completa con la fortaleza de la esencia de esa criatura. Adquirir comprensión de estos hermanos y hermanas es un proceso de curación, y debe ser abordado con humildad e intuición"

Jamie Sams y David Carson (1992)

\section{Consideraciones Generales}

En la actualidad se observa un notable interés por la incorporación de animales en diferentes disciplinas relacionadas con el trabajo terapéutico en el ámbito de la salud humana, por lo que este tema se ha convertido en foco de numerosas investigaciones científicas.

Autores como Chandler (2005), citado por Maestre (2015a, pág. 4), establece que "las Terapias Asistidas con Animales se están desarrollando notablemente como actividad terapéutica, ofreciendo una interacción positiva entre el humano y el animal, facilitando su recuperación y mejora de la actitud y actividad personal".

Según Mendoza (2017a, pág. 4), "el vínculo humano-animal puede traer muchos beneficios medibles, ayudando a controlar los niveles de depresión, reducción de estrés, aliviar el sufrimiento y la ansiedad, entre otros".

De la misma forma, autores como Tsai, Friedmann y Thomas (2010); Johnson, Meadows, Haubner y Sevedge (2008), citados por Mendoza (2017b, pág. 4), destacan los beneficios de la compañía de animales "como un recurso para afrontar enfermedades crónicas y su tratamiento".

En la actualidad poco se discuten las utilidades que supone la incorporación de animales en los protocolos de atención de personas con discapacidad, así como en programas dirigidos a sectores que requieren una ayuda especial, como puede ser la población penitenciaria, ancianos, niños en zonas urbanas deprimidas o con dificultades de aprendizaje. 


\section{Aproximación conceptual}

Teniendo como referencia la definición aportada por Fredrickson (1992): se describirán someramente en este apartado las diferentes intervenciones que se realizan con animales, entre las que se pueden mencionar: terapia asistida con animales, actividades asistidas con animales y educación asistida con animales.

En la actualidad son varias las definiciones empleadas para conceptuar las Terapias Asistidas con Animales. En este sentido, se hará referencia a la expuesta por Tucker (2004), citado por Robles, (2015, pág. 17), quien define este tipo de intervención como "una modalidad de tratamiento terapéutico en la que un animal, el cual cumple determinados criterios, forma parte integral del proceso de tratamiento".

En relación con las Actividades asistidas con animales, estas van dirigidas a fomentar la motivación, la educación y la recreación, con el fin de incrementar la calidad de vida de los destinatarios. Según Maestre (2015b, pág. 8), "se desarrollan en entornos con unos planteamientos más lúdicos y el encuentro con el animal es más espontáneo".

Y en la Educación asistida por animales, se persigue una "intervención dirigida por un profesional de la educación, en la que participa un animal entrenado para la consecución de unos objetivos pedagógicos o para la estimulación de diferentes áreas de desarrollo". (Maestre, 2015c).

\section{Tipos de Animales que se usan en la Terapia con Animales}

Sobre este particular, Fine (2003a): sostiene que entre los animales que se emplean con más frecuencia se encuentran perros, gatos, caballos y delfines, estableciéndose la idoneidad del animal en función de las condiciones físicas y psicológicas de los usuarios, así como también del tipo de centro en el cual se realizará la intervención. El perro, es el más utilizado fundamentalmente por la variedad de razas, temperamento y facilidad de 
adiestramiento. Se emplean además pájaros, mamíferos, acuarios con peces en las salas de espera de algunos consultorios odontológicos, para producir un efecto relajante en los pacientes.

\subsection{A continuación, se describirán brevemente las terapias más utilizadas:}

3.1.1. Terapia Asistida con Perros: es una técnica basada en el contacto cercano con perros entrenados, que intervienen dentro de un programa terapéutico con un objetivo definido de mejora intencionado, donde se persigue la participación de los pacientes en diferentes actividades sensoriales y perceptivas.

3.1.2. Terapia Asistida con Delfines: es una terapia en la cual participan delfines, así con la ayuda de un terapeuta se realiza un procedimiento dirigido al paciente, dentro y fuera del agua, con el propósito de mejorar algunas condiciones físicas y mentales. Puede ser aplicada a personas de diferentes edades, el tiempo recomendado es por un período de quince días con sesiones entre 35 a 45 minutos, donde las personas interactúan a través del nado, el juego y las caricias.

3.1.3. Terapia Asistida con Caballos: ha sido definida como una forma especializada de terapia física que utiliza equinos para tratar a personas con trastornos del movimiento, asociados a varias afecciones neurológicas y neuromusculares. Esta terapia a su vez se clasifica en dos modalidades diferentes: Hipoterapia y Equitación Terapéutica.

Es importante resaltar que no existe un animal específico para cada tratamiento, y que el terapeuta utiliza la motivación del animal para trabajar objetivos determinados, pero sí que es cierto que hay algunos animales con los que es más fácil trabajar unas cosas u otras. 


\section{Contextos de Aplicación}

En función de las necesidades y características de los usuarios, existen variados centros donde desarrollar la terapia asistida con animales. En este sentido, se pueden encontrar en la literatura revisada diversas clasificaciones sobre estos contextos. En este trabajo se tomarán las propuestas por Fine (2003b), y Gunter (2002), citados por Maestre, (2015d, pág. 13), los cuales las engloban de la siguiente manera: Centros para personas con trastornos del desarrollo, Centros Educativos, Programas con animales para Servicios y Programas Residenciales Basados en Instituciones, éstos se describirán a continuación.

En cuanto a los Centros para personas con trastornos del desarrollo, "se engloban actividades de toma de contacto físico, de la enseñanza de la responsabilidad de autocuidado y del cuidado del animal, tareas de estimulación y situaciones de ocio". (Maestre, 2015e, pág. 13).

En los Centros Educativos, son múltiples las actividades que pueden realizarse con compañía de animales, ya que "pueden enseñarse habilidades cognitivas, ayudar a que los niños mejoren sus capacidades motoras, utilizarlos para mejorar la lectura y el rendimiento académico, fomentar la socialización, potenciando conductas prosociales y resolución de conflictos". (Maestre, 2015f, pág. 14).

Con respecto a los Programas con animales para Servicios, en estas intervenciones "el animal que se utiliza por excelencia es un perro guía que cumple unos criterios específicos para el tratamiento y ha sido entrenado previamente para su cometido". (Maestre, 2015g, pág. 14).

Por su parte, en los Programas Residenciales Basados en Instituciones, estas intervenciones se ponen en práctica en centros donde se ofrecen cuidados por largos períodos, como pueden ser residencias de personas mayores, hospitales y centros penitenciarios. 


\section{Beneficios de la Intervención con animales}

Los beneficios que se obtienen en las Intervenciones asistidas con animales se dividen principalmente en cuatro grandes áreas: físicos, psicológicos, educativos y sociales.

En este sentido, Wilson y Turner (1998), citados por Maestre, (2015h, pág. 10), exponen que "la presencia de un animal de compañía ayuda a prevenir la aparición de diferentes tipos de enfermedad, facilita su afrontamiento y a su vez, favorece la rehabilitación de la misma".

En esta misma línea, Maestre, (2015i, pág. 11), afirma que esta modalidad de tratamiento terapéutico:

Está diseñada para promover las mejoras en el funcionamiento físico como habilidades motoras, equilibrio y balance, relajación, entre otras, en la parte cognitiva ayuda a estimular la atención, percepción, memoria, lenguaje, praxis y mejora la atención. Por el lado educacional aporta a la retención de memoria a largo o corto plazo, conocimiento de conceptos, vocabulario y comunicación. Además, esta modalidad terapéutica sirve como motivación para el paciente a realizar la actividad previamente planificada por el psicólogo o terapeuta, permite la interacción entre personas, aumenta la confianza y autoestima y disminuye los sentimientos de soledad.

\section{Del animal guía o de terapia}

En relación a la concepción de los animales, existen diversas posturas, a saber: mecanicista; (los animales son seres autómatas, sin almas, ni mente, no razonan y por ende no tienen la capacidad de sufrir ni sentir), utilitarista; (el padecimiento, es el que otorga a un ser vivo el derecho a ser considerado moralmente y consecuencialmente a no sufrir), consecuencialismo; (toda acción genera consecuencias y ello permite distinguir el bien y el mal), especismo; (que distingue al ser humano de otras especies), contractualista; (no causar sufrimientos innecesarios a los animales constituye un deber), igualitarista o animalista; (propone la igualdad entre animales, humanos o no). 
Corresponde reflexionar luego de discernir sobre los beneficios de estas terapias con animales, respecto a la postura de éstos y su participación dentro de los referidos procesos.

\section{El animal no es un simple recurso dentro de la terapia}

El primer aporte que se considera necesario hacer, guarda relación a la concepción del animal dentro de la planificación de la terapia, es decir, realmente el animal puede ser considerado un material o recurso más, dentro de tal programación, como un lápiz, una almohada o una colchoneta. Revisando algunas planificaciones de terapias asistidas para niños con autismo y parálisis cerebral, puede verse como se cataloga al perro que participa en la terapia como un recurso, luego del cual se enumera: comida para perro, cepillos, colchonetas. Se estima que tal etiqueta, no sólo es absurda ante el desarrollo de la terapia, sino que además sesga la planificación, logrando invalidarla, y se detallará por qué:

Supóngase que se programan terapias asistidas con un perro Golden Retriever o Labrador Dorado, la planificación debe incluir la aparición de algunos factores que puede presentar el can, los cuales pueden ser previos y otros pudieran producirse de manera imprevisible durante la terapia. Se pudiera mencionar como factor previo el estrés, cansancio, sueño, o calor, lo cual debe ser detectado por el terapeuta y no se le debe restar importancia, ya que la condición física del animal debe ser óptima, no sólo para que la terapia pueda ser efectiva, sino porque se trata de un ser vivo que requiere atención, máximo cuando se está obteniendo de él un provecho económico.

La planificación debe prever algunos factores que pudieran aparecer al momento de la terapia, relacionados con los movimientos involuntarios del paciente, el número de pacientes, la temperatura del lugar, la exigencia física que se le imponga al animal, cuando ha de caminar o correr como parte de la terapia. Por ello el terapeuta, si bien debe atender al paciente como agente 
que recibe el beneficio de la terapia, también deberá estar alerta de cualquier signo que pudiera manifestar el animal, recordando que aun cuando ha recibido un entrenamiento y ha pasado por un riguroso proceso de selección, es inevitable que ante algunas situaciones reaccione instintivamente.

El animal no puede ser sobre exigido en cuanto al número de sesiones en que participará, ni puede tratarse de sesiones extenuantes, el Task Force for IAHAIO (2014), recomienda que una terapia asistida con animales puede tener una duración de 30 a 45 minutos, dependiendo de la cantidad de personas que participen en casos de terapias grupales, sugiriendo siempre que en caso de que el terapeuta observe signos de cansancio o aburrimiento en el animal debe detener la sesión o realizar un break. Asimismo, recomiendan que el can participe como máximo en 3 sesiones a la semana.

Lo antes descrito muestra, los diversos factores que pueden incidir para la planificación, desarrollo, continuidad o suspensión de una terapia con animales, los cuales de no ser tomados en cuenta por el terapista constituirían una novatada que pudiera generar consecuencias desastrosas. Los profesionales realmente expertos en terapias con animales atienden frecuentemente al animal, chequean periódicamente su salud y están atentos a cualquier signo o síntoma particular que presente el mismo, sin saturar al animal con extenuantes y excesivas sesiones, igualmente están en el deber de informar a los pacientes y sus familiares las condiciones de la terapia y los motivos por los cuales pudiera suspenderse, posponerse o prorrogarse.

De esta manera, se precisa reconsiderar al animal que participa en la terapia, para que no sea etiquetado como un recurso, en sintonía con su entidad como ser vivo, en respeto por sus derechos, tomando en cuenta lo previsto en la Declaración Universal de los Derechos del Animal (1977a), en su artículo 7 que dispone: "Todo animal de trabajo tienen derecho a una limitación razonable del tiempo e intensidad de trabajo (...) y al reposo".

Más allá de lo anterior, si se reflexiona realmente en la terapia con 
animales, independientemente que el objetivo lo marque el terapeuta, la función sanadora y reparadora del humano se centra fundamentalmente en el animal, quien realmente produce una transformación en el paciente, de allí la gran importancia que posee este animal en dicho proceso.

\section{Respetar los procesos biológicos del animal}

El animal posee necesidades biológicas y fisiológicas, que deben ser respetadas, por ello, no puede someterse a horarios o sesiones extenuantes que impidan su natural desenvolvimiento, a fin de cubrir requerimientos terapéuticos de humanos.

En este sentido, el caso de canes que participan en terapias, tienen derecho a realizar sus necesidades con naturalidad, se le debe tener agua siempre disponible durante el transcurso de la misma, igualmente debe ser alimentado con una dieta acorde a su raza, tamaño y corpulencia, de tal manera que pueda recuperar las energías consumidas, manteniendo un peso corporal idóneo, debe respetarse un tiempo de descanso suficiente, para que el sueño resulte reparador, evitando el uso de fármacos o drogas en procura de mejores resultados o estimular interacción.

Es preciso acotar que la Declaración Universal de los Derechos del Animal (1977b), en su artículo 2, el literal a, establece que: "a). Todo animal tiene derecho a ser respetado...", asimismo el mismo artículo en su literal c, dispone: “...c). Todos los animales tienen derecho a la atención, a los cuidados y a la protección del hombre". Y el artículo 7 dispone: "Todo animal de trabajo tienen derecho $(. .$.$) a una alimentación reparadora..."$

Se hace necesario referirse de manera específica a la delfinoterapia, como una de la terapias con animales más atacadas por los activistas y animalistas, precisamente por no respetar los procesos biológicos naturales de los cetáceos, partiendo de apartarlo de su hábitat natural para ponerlo en cautiverio, así como las experiencias que se han tenido en diversas partes del 
mundo, con muertes de delfines sometidos a excesos y estrés, así como por las condiciones críticas del cautiverio y excesivos traslados.

En una investigación realizada por Castello, Alaniz y Vega (2007a), en la que se profundiza con relación a la delfinoterapia se analizó que:

Los delfines son los mamíferos más inteligentes de la vida oceánica y toda su conducta se encuentra determinada por millones de años de evolución en su medio natural. Toda esta conducta queda cancelada y suprimida desde el momento de la captura de un individuo de la manada, que además rompe la organización del grupo, dando como resultado un daño cualicuantitativo de los cetáceos, tanto en lo general (grupo), como en lo particular (individuo). (pág. 33).

Es así como, resulta en extremo importante para los cetáceos mantenerse organizados en manadas. En la misma investigación Castello, Alaniz y Vega (2007b), concluyeron que:

Los datos reales del cautiverio, como son la forma de captura, las condiciones insalubres y artificiales de los estanques de concreto y lo agresivo de las medidas de tratamiento del agua que repercute en el daño ocular y dérmico de los animales, así como las condiciones del llamado Condicionamiento Operante (entrenamiento), que no es más que el aprendizaje a base de hambre, son ocultadas a espectadores. (pág. 33).

En ese reporte, también se publican cifras de alta mortalidad de cetáceos, igualmente se hace alusión a un conjunto de muertes que no son registradas, y que generalmente se producen al momento de realizar las capturas, momentos en que se generan lesiones y accidentes, por ello afirman Castello, Alaniz y Vega (2007c): que ello permite "sospechar un enorme daño estructural y social de las manadas; así, como sufrimiento de los individuos, lo que define al cautiverio como una industria netamente cruel para el individuo y altamente impactante para el ecosistema". (pág. 33).

De allí que, en atención a la investigación mencionada, la situación de los delfines en cautiverio incluso para fines terapéuticos contraviene lo 
dispuesto en el artículo 4 de la Declaración Universal de los Derechos del Animal (1977c), que establece: "a). Todo animal perteneciente a una especie salvaje, tiene derecho a vivir en libertad en su propio ambiente natural terrestre, aéreo o acuático y a reproducirse. b). Toda privación de libertad, incluso aquella que tenga fines educativos, es contraria a este derecho".

La máxima crítica que se hace a la delfinoterapia es la falta de sustentación científica de los beneficios y mejorías, lo que hace infructífero y estéril el daño que con el cautiverio se causa al delfín atrapado y a la manada o grupo del cual forma parte. Claro está, los promotores de esta terapia, dueños de acuarios y delfinarios, incluso algunos veterinarios proteccionistas coinciden en enunciar un conjunto de beneficios en humanos, especialmente relacionados con algunas patologías o condiciones.

Sobre este particular, una investigación anónima denominada "Parque Delfinoterapia de Pirque" (1998), citada por Castello, Alaniz y Vega (2007d), en el aspecto metodológico de dicho proyecto se menciona que:

...después de 8 sesiones con niños, jóvenes y adultos se han logrado los siguientes avances porcentuales en las siguientes patologías que se mencionan: Retraso psicomotor (RPM), 70\%; parálisis infantil (PC), 70\%; Autismo $65 \%$; Crisis convulsivas $85 \%$; Hemiplejía 75\%; Síndrome de Down 85\%. En otras 12 enfermedades neurológicas se mencionan avances que varían entre el 50 y el $90 \%$. Falta mencionar que criterios evaluativos y/o pruebas, o metodología diagnóstica se utilizó para cuantificar los avances mencionados, sobre todo en aquellas patologías irreversibles, por ej. Síndrome de Down, Microcefalia y Lesión Cortical. (pág. 31).

Más recientemente la historia del entrenador de delfines José Luis Barbero, sacudió las redes y los diarios locales, luego de que se suicidara tras la publicación de un video donde se veía parte de los entrenamientos, en los que se reunieron algunas escenas de maltrato, la audiencia se dividió entre los proteccionistas y animalistas que repudiaban las prácticas del entrenador, 
mientras que el otro grupo lamentaba el fallecimiento del humano y culpaba a la jauría animalista de haber provocado aquel fatal desenlace, con un video supuestamente alterado y editado, destacando la noble labor social que se llevaba a cabo en aquel acuario de Mallorca, España.

Ciertamente este trabajo no pretende aclarar los detalles de aquel fatídico siniestro ocurrido en el 2015, pero sí permite reflexionar, sobre las prácticas terapéuticas con delfines, surgiendo las siguientes interrogantes: ¿Cómo es el proceso de entrenamiento de los delfines?, ¿El reforzamiento y condicionamiento de los delfines conlleva castigos corporales?, ¿Son idóneas las condiciones de cautiverio del delfín en parques, acuarios y zoológicos?, ¿Existen estadísticas y reportes de las muertes del delfines en cautividad y sus causas?, ¿De existir estadísticas, cual es la incidencia de muertes de delfines por negligencia, impericia, enfermedad, estrés y otras implicaciones psicológicas?, ¿Es justificable el costo-beneficio, en relación a delfines en cautividad, versus beneficios de las terapias en humanos?

La documentación sobre el tema permite alertar sobre el grave daño ecológico que se causa con el cautiverio de delfines, cuya especie se encuentra cada vez más amenazada, lo que constituye un problema de afectación de la biodiversidad. Por otro lado, los efectos nocivos en delfines incluso nacidos en cautiverio son letales, de hecho, animalistas advierten que la memoria genética en animales salvajes no se pierde en una, ni 50 generaciones, se requieren siglos. De allí que, ni la aparente sonrisa de algunas especies de delfines puedan ocultar la depresión y tristeza que pueden vivir estos animales en cautividad.

A este respecto, Low (2012): en la Declaración de Cambridge sobre la Conciencia indicó que: "los seres humanos no son los únicos que poseen los sustratos neurológicos necesarios para generar conciencia. Animales no humanos, incluyendo todos los mamíferos y pájaros, y muchas otras criaturas, incluyendo los pulpos, también poseen estos sustratos neurológicos." (pág. 3). 
Ante la publicación de la referida Declaración de Conciencia se ha comenzado a discutir que los cetáceos son merecedores de derechos humanos, toda vez que poseen conciencia, lo que se creía un atributo exclusivo del humano, de allí que se vete su caza o cautividad.

\section{Brindar afecto al animal}

Es importante que el animal que participa en terapias asistidas reciba afecto tanto del terapeuta como en la medida de las posibilidades del paciente y sus familiares. Este afecto que se expresa con caricias, mimos, lenguaje hablado y corporal, facilita el contacto con el paciente y hace que el clima de la terapia sea propicio para la sanación.

Normalmente esta afinidad y complicidad entre el animal y el terapeuta van surgiendo espontáneamente, dado el trato e interacción continua. Sobre todo, gracias al animal que posee cualidades sanadoras y terapéuticas innatas, que produce en los humanos la necesidad de demostrarle amor.

Como muestra de lo anterior, se ha expuesto que niños incapaces de mostrar empatía incluso hacia sus padres, por diversos motivos, logran demostrar su afecto hacia los animales de terapia, acostándose sobre ellos, abrazándoles y hasta acariciándoles. Se trata de uno de los beneficios más presentes en estas terapias, y que causa impresión y alegría en los padres y familiares, que por primera vez ven a su hijo demostrar afecto y empatía, transmitiendo así una sensación de sanación y sosiego también a los familiares del paciente, que crecen y sanan conforme avanzan las terapias.

En pacientes agresivos, que se auto flagelan, se ha observado como el animal logra con su interacción cesar la conducta hostil del paciente, llevándolo a la calma. Los canes lo hacen abarcando al humano y lamiéndole, buscando que sienta su cercanía y calor, hasta que el paciente detiene la auto agresión y culmina por abrazarlo. Estos resultados son poco obtenidos por otro humano, por más amor que sienta hacia el paciente. 
Es preciso acotar que, en ocasiones se crea un nexo de dependencia entre pacientes y animales, y en caso de que se suspendan las terapias, o se cambie el animal, podría experimentarse un retroceso o involución. También ocurre que cuando animales guías mueren o son apartados del paciente, puede producirse cuadros depresivos que afectarán la condición previa del paciente. Por estos motivos el terapeuta puede sugerir el cambio constante del animal de asistencia, incluso el cambio de terapia. También puede ocurrir que el paciente no tolere o resista el contacto con el animal, por predisposiciones psicológicas o por la misma condición que padezca, por ello es necesario alertar y reconocer que un animal de servicio no es para todos.

En definitiva, es importante que el animal de asistencia o guía reciba cariño y compasión de parte de todos los sujetos que intervienen en los procesos, debiendo retirar al animal del contacto con cualquier persona que en algún momento reaccione agresivamente o lo maltrate. En este sentido, la Declaración Universal de los Derechos del Animal (1977d), en su artículo 3 establece que: "a). Ningún animal será sometido a malos tratos ni a actos crueles". El cariño constituye pues, una forma de reforzamiento positivo para los animales de servicio, que deben ser recompensados, apropósito que no reciben remuneración, la única manera de retribuirles es con cuidos y afecto.

\section{Supervisar los entrenamientos}

Muchas han sido las denuncias y videos que acaparan la atención en el Internet, en las que se discute y observan maltratos de animales, también es cierto que la mayoría se suscitan en Circos, Acuarios y Zoológicos, aunque algunas terapias animales no escapan de estos señalamientos. Por ello, es importante velar por los derechos del animal en las fases de entrenamiento, pues pudiera ser la más crítica en cuanto a maltrato animal se refiere.

La supervisión de los entrenamientos puede hacerse a través del trabajo conjunto de entrenadores, de modo que uno controle al otro, o por 
medio de cámaras que registren de modo integral dichos entrenamientos, a las que tenga acceso el propietario del centro o institución que presta el servicio.

Conocer cómo se entrenan los animales guías o de asistencia, resultará muy beneficioso para quien contrata el servicio, pues sí el animal no sufre malos tratos, podrá disfrutar de la terapia para sí o su familia, sin culpabilidad, a sabiendas que aquel animal hacia quien seguramente despertará sentimientos de cariño y amistad, no ha sido previamente agredido; por el contrario, si el paciente o algún familiar descubre o percibe que el entrenamiento se ha hecho por medio de castigos corporales o psicológicos, se cuestionará sí debe continuar la terapia o sí es mejor no contribuir con estos episodios.

En caso de maltratos de canes, es difícil evitar reconocerlos, pues el animal dará señales inequívocas frente a su entrenador, por lo que tarde o temprano el paciente y sus familiares, reaccionarán o naturalizarán la violencia animal. En casos de terapias con delfines, se dificulta constatar estos malos tratos, sobre todo, por la sonrisa típica del delfín, que guarda relación directa con su morfología, y no con un sentimiento, aunado a que las señales que muestra, de alejarse nadando o hundirse al fondo de los estanques, incluso sus quejidos y llantos, no son a menudo interpretadas por los pacientes o sus familiares. En algunos casos de terapias con equinos, el entrenador utiliza una vara en su mano derecha, con la que aduce estimular al caballo, pero en realidad, es un instrumento de dominación que le infringe dolor, por otro lado son utilizados como tracción a sangre, lo cual ya de por sí ha sido objeto de críticas por los animalistas, esto sin mencionar herraduras, gríngolas y látigos, más comunes en otras prácticas con equinos, pero que deben ser objeto de reflexión, a fin de no naturalizar el maltrato animal. 


\section{No naturalizar el maltrato animal}

Aquellos pacientes y familiares que acudan a terapias con animales, deben tener presente que el maltrato animal no es tolerable. El entrenamiento debe hacerse con técnicas que garanticen un condicionamiento libre de castigos corporales o psicológicos, para ello los entrenadores deben actuar sin apremios, con la paciencia característica relatada en la literatura, hacia animales no humanos.

Los pacientes o familiares que sospechen o hayan evidenciado un maltrato animal deben denunciarlo, para ello existen un sin número de asociaciones de protección animal, ONG, grupos animalistas, en algunos países unidades de gestión animal y en otros jueces agrarios con competencia en biodiversidad, fauna doméstica y silvestre. Tener contacto con este sistema de protección animal, es bastante sencillo, pues suelen acaparar las redes sociales, lo importante es informarse previamente sobre la fama del organismo antes de acudir a ellos. Lo lógico es que estas organizaciones antes de difundir información sobre maltrato por parte de algún centro de terapias animales, realicen una investigación previa, conversen con los directivos, constaten información, inspeccionen el centro con la participación de veterinarios, todo ello es necesario para lograr un panorama claro del posible maltrato animal y para arribar a conclusiones.

Denunciar los maltratos con animales, forma parte de una estrategia de coerción hacia centros de terapias con animales, que propenderá a una cultura de respeto hacia estos. Presenciar y tolerar el maltrato animal, te convierte en cómplice, lo que más tarde o más temprano puede ser incluso castigado. No en vano la Declaración Universal de los Derechos del Animal (1977e), en su artículo 14 establece que: "a). Los organismos de protección y salvaguarda de los animales deben ser representados a nivel gubernamental. b). Los derechos del animal deben ser defendidos por la ley, al igual que los derechos del hombre". 


\section{Consideraciones Finales}

Las terapias asistidas con animales, los animales guías y la educación con animales, son prácticas que están siendo cada día más difundidas, el animal proporciona y contagia una sensación innegable de paz y equilibrio con la naturaleza y contribuye en gran medida en la salud de los seres humanos, mejorando su calidad de vida, la polémica radica en que estos animales que participan en terapias no pueden ser maltratados, pues lo que también ha avanzado es la cultura de protección animal.

De esta forma, en este documento se han enumerado algunas claves para que el trato de animales de asistencia sea armónico y amigable, entre ellas: entender que el animal no es un simple recurso dentro de la terapia, respetar los procesos biológicos del animal, brindar afecto al animal, supervisar los entrenamientos, no naturalizar el maltrato animal, todo ello descansando sobre la Declaración Universal de los Derechos del Animal (1977f), que constituye un referente global, pero al cual cada día se le adicionan leyes, normativas y ordenanzas internas, en diversas latitudes, que se orientan hacia la protección de estos hermanos animales.

\section{Referencias}

Castello, H., Alaniz, Y., \& Vega, C. (2000a,b,c,d). Los delfinarios en México. Un Informe Crítico. México, D.F.: Conservación de Mamíferos Marinos de México. Recuperado de:

http://www.academia.edu/4388371/Los delfinarios en M\%C3\%A9xic

o. Un informe cr\%C3\%ADtico

Chandler, C. (2005). Animal Assisted Therapy in Counseling. New York, US: Routledge.

Declaración Universal de los Derechos del Animal (1977a,b,c,d,e,f). Adoptada por la Liga Internacional de los Derechos del Animal y por las Ligas Nacionales afiliadas tras la 3 o Reunión sobre los derechos del 
Animal. Londres: Proclamada ante la ONU 1978, págs. 21-23.

Fine, A. $(2003 a, b)$. Manual de terapia asistida por animales: Fundamentos teóricos y modelos prácticos. Barcelona, España: Fondo Editorial de la Fundación Affinity.

Fredrickson, M. (1992). Handbookfor animal-assisted activities and animal-assisted therapy. Renton, WA: Delta Society.

Gunter, B. (2002). Animales domésticos: Psicología de sus dueños. Barcelona, España: Paidós.

Johnson, R., Meadows, R., Haubner, J., \& Sevedge, K. (2008). Animalassisted activity among patients with cancer: effects on mood, fatigue, self-perceived health, and sense of coherence. Oncol Nurs Forum, 35(2), pp. 225-232. Recuperado de:

https://doi.org/10.1188/08.ONF.225-232

Low, P. (2012). The Cambridge Declaration on Consciousness. Francis Crick Memorial Conference on Consciousness in Human and nonHuman Animals. Inglaterra: Churchill College, University of Cambridge.

Maestre, S. (2015a,b,c,e,d,f,g,h,i). Terapia asistida con animales: Propuesta de abordaje en una unidad de convivencia desde la educación social. Trabajo de Fin de Grado. Educación Social. España: Universidad de Valladolid. Recuperado de:

https://uvadoc.uva.es/bitstream/10324/14444/1/TFG-G\%201305.pdf

Mendoza, B. (2017a,b). Aplicación interactiva para la Terapia Asistida con Perros para los pacientes del Centro Canoterapia Ecuador. Tesis de Pregrado. Ecuador: Universidad Católica de Santiago de Guayaquil. Recuperado de:

http://www.bibliotecasdelecuador.com/Record/ir-:33178276/Description

Robles, L. (2015). Disminución de los niveles de ansiedad en adultos mayores institucionalizados a través de actividades asistidas con 
animales. Trabajo de Grado de Maestría. México: Universidad Autónoma de Nuevo León. Recuperado de: http://eprints.uanl.mx/9683/ Sams, J., \& Carson, D. (1992). Medicine cards: The discovery of power through the ways of animals. pp. 224. ISBN: 093968053X, ISBN13: 9780939680535. U.S.: Bear \& Company (first published September 1988).

Task Force for IAHAIO (2014). The IAHAIO definitions for animals assisted interventions and animal assisted activity and guidelines for wellness for animals involved. USA: IAHAIO White Paper, pp. 1-10. Recuperado de:

http://iahaio.org/wp/wp-content/uploads/2017/05/iahaio-white-paperfinal-nov-24-2014.pdf

Tsai, C., Friedmann, E., \& Thomas, S. (2010). The effect of animal-assisted therapy on stress responses in hospitalized children. Anthrozoos. 23(3), 245-258. Recuperado de:

https://doi.org/10.2752/175303710X12750451258977

Tucker, M. (2004). The Pet Partners Team Training Course Manual. Bellevue, WA: Delta Society.

Wilson, C., \& Turner, D. (1998). Companion Animals in Human Health. Thousand Oaks. California, Estados Unidos: Sage. 


\section{Camilo Chacón Herrera}

e-mail: camilochacon2008@gmail.com

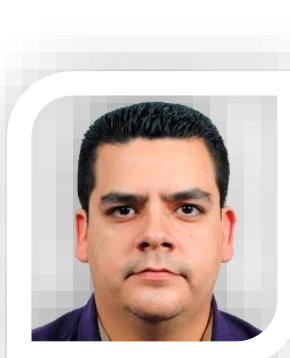

Nacido en Maracay, Venezuela. Abogado por la Universidad de Carabobo, especialista en Derecho Procesal Civil, con estudios en Derechos Humanos, Constitucional y Epistemología, con 10 años de experiencia docente en pre y postgrado en diversas Universidades de su país. Actualmente se desempeña como Juez Superior Agrario de los estados Aragua y Carabobo en Venezuela. 


\section{Marian Serradas Fonseca}

e-mail: mserradas@hotmail.com

Nacida en San Felipe, Venezuela. Doctora por la Universidad de Salamanca en el Programa Doctoral: "Avances y Perspectivas en Investigación sobre Personas con Discapacidad". Máster en Ciencia, Tecnología y Sociedad: Cultura y Comunicación en Ciencia y Tecnología. Especialista en Telemática e Informática en Educación a Distancia. Licenciada en Ciencias de la Educación, Mención Educación Especial. Actualmente se desempeña como Asesora Académica del Área Dificultades de Aprendizaje y Responsable de la Unidad de Investigaciones y Postgrado de la Universidad Nacional Abierta, Centro Local Yaracuy.

El contenido de este manuscrito se difunde bajo una Licencia de Creative Commons Reconocimiento- 